\title{
Cross sectional echocardiography for recognition of ventricular topology in atrioventricular septal defect
}

\author{
JULENE S CARVALHO, MICHAEL L RIGBY, ELLIOT A SHINEBOURNE, \\ ROBERT H ANDERSON
}

From the Department of Paediatric Cardiology, Brompton Hospital, London

SUMMARY The cross sectional echocardiograms of 150 children with atrioventricular septal defects were reviewed to determine the spatial orientation of the ventricles. In 125 cases with usual atrial arrangement (situs solitus), ventricular topology showed the right hand pattern. Of the 25 patients with atrial isomerism, 13 had similar right hand topology but $12(48 \%)$ had the left hand pattern. This finding was more common in hearts in the right side of the chest (dextrocardia), but there was no significant correlation between ventricular topology and number of atrioventricular valves or the ventriculoarterial connection. In part because of inferences for disposition of the conducting tissue, knowledge of ventricular topology is important when operation for atrioventricular septal defects is considered.

Ventricular topology can be promptly recognised by cross sectional echocardiography.

For hearts with both morphologically right and left ventricles, Van Praagh and his colleagues introduced the concept of right and left hand spatial orientation of the ventricles. ${ }^{1}$ This concept is particularly important in describing hearts with an atrioventricular septal defect and atrial isomerism because the spatial orientation of the ventricles cannot be inferred from the category of atrioventricular connection.

Topology or topographical anatomy is the science of the description of a part of the body in terms of where it lies and its surrounding structures. In socalled right hand topology, it is the palmar surface of the observer's right hand that can be placed on the septal surface of the morphologically right ventricle, with the thumb in the tricuspid valve, the wrist in the apical trabecular component, and the fingers in the outlet. Such an arrangement is found in the normal heart. Left hand pattern topology, the mirror image arrangement, is characteristically found in hearts with congenitally corrected transposition with the usual atrial arrangement.

\section{Patients and methods}

One hundred and fifty consecutive cases of atrioventricular septal defects investigated echocardiographically between January 1983 and September

Requests for reprints to Dr Julene S Carvalho, Department of Paediatric Cardiology, Brompton Hospital, Fulham Road, London SW3 6HP.

Accepted for publication 15 September 1988
1986 were reviewed retrospectively. The atrial arrangement (situs) was inferred from analysis in short and long axis views of the abdominal great vessels. Cases with usual atrial arrangement (situs solitus) showed a left sided aorta and a right sided inferior vena cava at the level of the diaphragm. Atrial isomerism was inferred echocardiographically from the arrangement of the aorta and major venous structures in the abdomen. Left atrial isomerism was judged to be present when there was a venous structure posterior to the usually midline abdominal aorta. This posterior venous stucture represented the azygous continuation of the inferior vena cava, and no normal inferior vena cava was seen. Right atrial isomerism was inferred when both the aorta and an anterior venous structure, shown to be the inferior vena cava in long axis view, were on the same side of the spine. ${ }^{2}$

Apical and subcostal four chamber sections were analysed to ascertain ventricular topology. Ventricles were identified as being of the right or left type on the basis of their anatomical features. In the morphologically right ventricle the atrioventricular valve shows chordal insertions into the ventricular septum (fig 1). The ventricle itself has an apical papillary muscle (fig 2), a septomarginal trabeculation (fig 3), and coarse trabeculations (fig 4). Conversely, the morphologically left ventricle was identified by absence of any chordal attachments to the septum (fig 1), the possession of characteristically paired papillary muscles, and a fine trabecular pattern (fig 

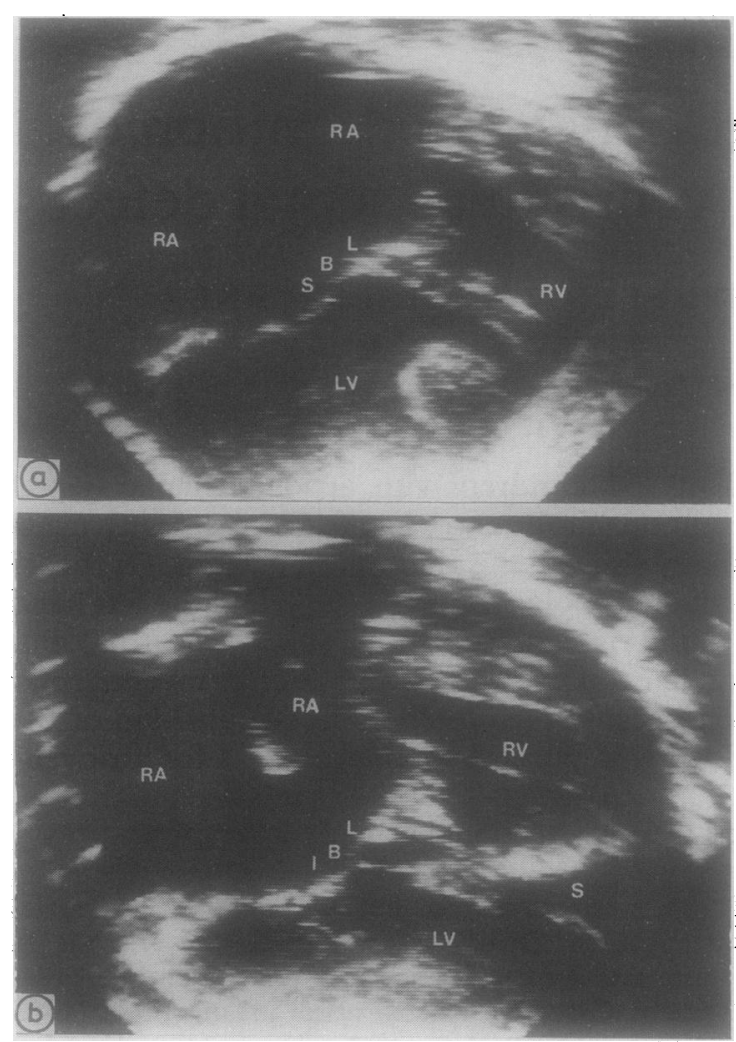

Fig 1 Atrioventricular septal defect with right atrial isomerism and left hand pattern topology. Note chordal insertions to the septum $(S)$. $R A$, right atrium; $R V$, right ventricle; $L V$, left ventricle; $S B L$, superior bridging leaflet; $I B L$, inferior bridging leaflet.

4). In patients with atrial isomerism, we identified the cardiac position within the chest, the presence of a common or two separate atrioventricular valves, and the ventriculoarterial connections. These features of the heart were correlated with the ventricular topology.

\section{Results}

\section{ATRIAL ARRANGEMENT}

Among 150 patients with atrioventricular septal defects, $125(83 \%)$ had lateralised atria, all with usual atrial arrangement. All were found to have right hand pattern ventricular topology (fig 4). The remaining 25 cases had atrial isomerism-13 had right and 12 left isomerism. In $13(52 \%)$ of them topology showed a right hand pattern (fig 5) and in the other 12 patients it showed a left hand pattern (fig 1). Right and left hand patterns were similarly distributed in the two subgroups with atrial isomerism. Fifty four per cent of patients with right and $50 \%$ of those with
Carvalho, Rigby, Shinebourne, Anderson $\underset{\frac{0}{T}}{\stackrel{\varpi}{\Phi}}$ left isomerism showed topology with a right hand pattern.

\section{CARDIAC POSITION}

A left sided heart was present in 11 patients, 9 of whom had the right hand topological pattern. Eleven $\overline{\frac{\sigma}{\omega}}$. patients had a right sided heart and nine of these had $\vec{\Phi}$ a left hand pattern topology. The different associa- $\varrho$ tions are unlikely to be due to chance ( $\chi^{2}$ with Yates' correction, $6.5 ; \mathrm{p}<0.05)$. The remaining three $\vec{\circ}$ patients had a heart in the middle of the chest, two of $\overrightarrow{ }$ which had the right hand pattern.

\section{MODE OF ATRIOVENTRICULAR CONNECTION}

The atrioventricular junction had a common atrioventricular valve in 16 patients -10 with left and ${ }_{i}^{i}$ six with right hand pattern topology. Connection was $O$ through two separate atrioventricular valves in the

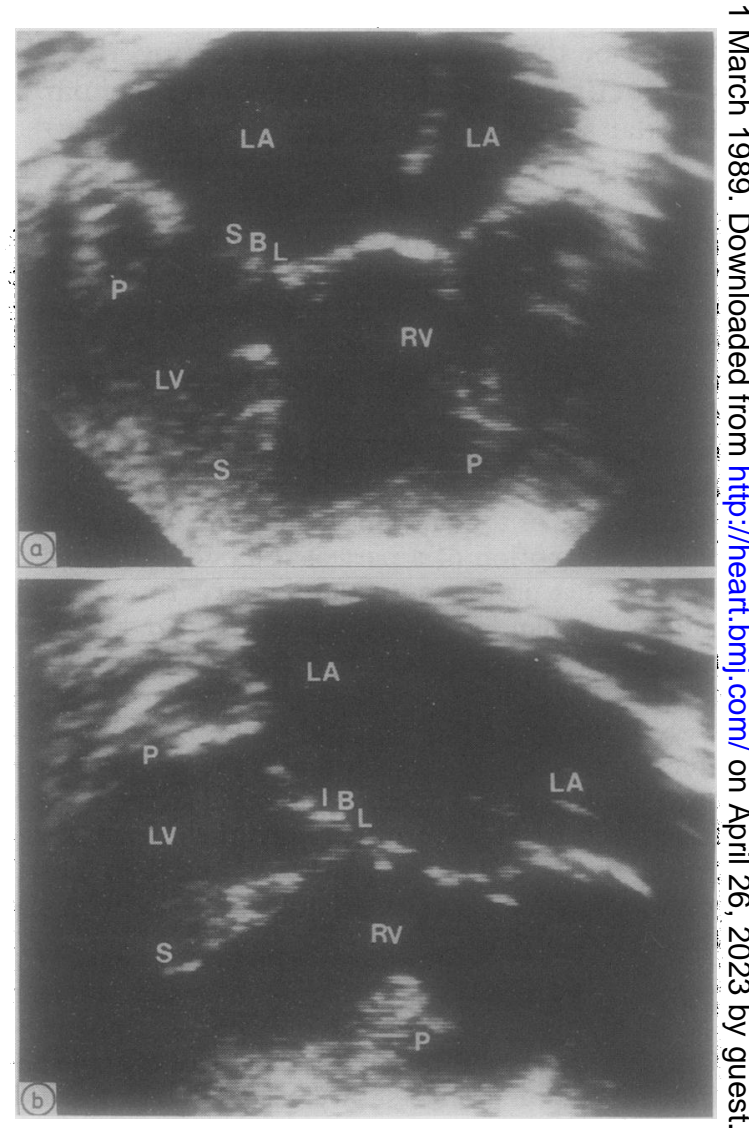

Fig 2 Atrioventricular septal defect with left atrial isomerism and left hand pattern topology. Note apical papillary muscle $(P)$ of the right ventricle $(R V)$. LA, left

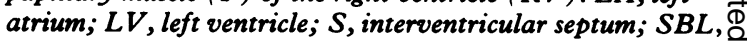
superior bridging leaflet; IBL, inferior bridging leaflet. 


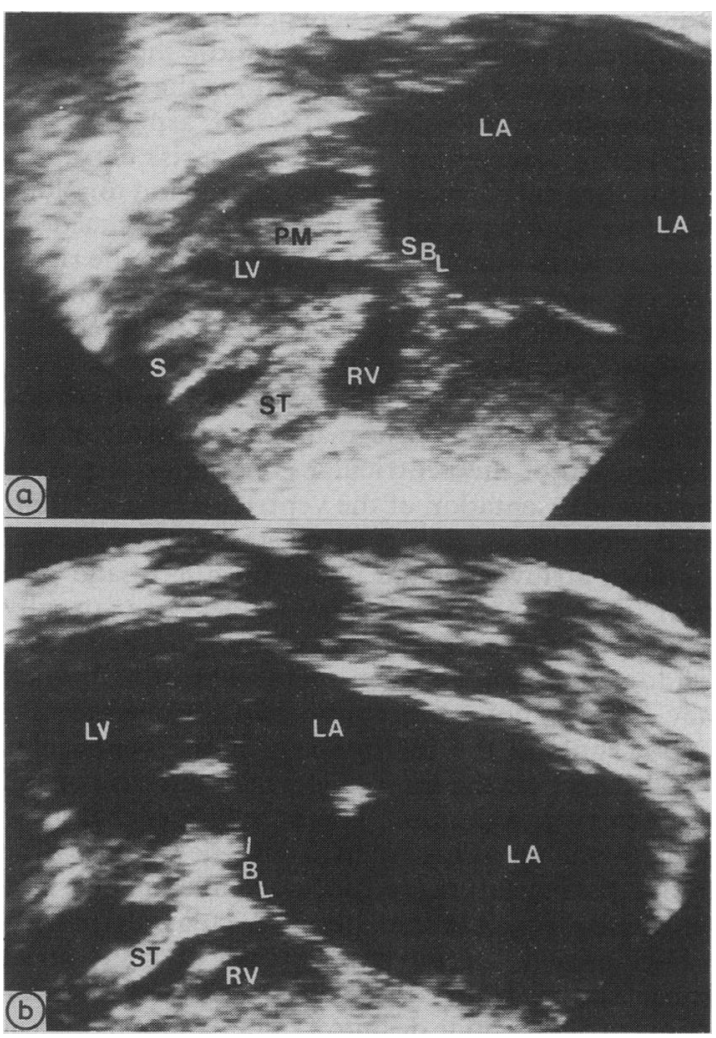

Fig 3 Atrioventricular septal defect with left atrial isomerism and left hand pattern topology. Note the septomarginal trabeculation ( $S T$ ) of the right ventricle $(R V)$ and the lateral papillary muscle (PM) of the left ventricle ( $L V)$. SBL, superior bridging leaflet; $I B L$, inferior bridging leaflet; $S$, interventricular septum; $L A$, left atrium.

remaining nine patients, seven of whom had a right hand topological pattern (fig 5). No significant difference was found between ventricular topology associated with the two modes of atrioventricular connections ( $\chi^{2}$ with Yates' correction, $2 \cdot 30, \mathrm{NS}$ ).

VENTRICULOARTERIAL CONNECTIONS

The ventriculoarterial connections were predominantly concordant (11 cases) and double outlet right ventricle ( 11 cases). In the first group, the right hand pattern was identified in seven patients. This pattern was also found in five patients in the second group.

\section{Discussion}

In our series of patients with an atrioventricular septal defect, all hearts with the usual atrial arrangement had ventricular topology with a right hand pattern. That was not the case in the presence of atrial isomerism, when the left hand pattern was found in about half of the cases. This applied when there were paired right atria or paired left atria. Thus while it is probable that atrial arrangement predicts ventricular topology when morphologically distinct left and right atria are present, atrial anatomy does not predict ventricular topology in the presence of atrial isomerism.

Right hand pattern topology was more common in left sided hearts as was the left hand pattern in right sided hearts. There is no significant correlation, however, between the mode of atrioventricular connection or ventriculoarterial connection and ventricular topology. Thus identification of ventricular morphology is the only reliable method to determine ventricular topology. Cross sectional echocardiography has proved to be a valuable and safe diagnostic tool in recognising the morphological features of each ventricle.

The concept of ventricular topology is important when surgical intervention is considered in patients with an atrioventricular septal defect because the distribution of the conduction system depends not only on the anatomical defect but also on topology. It is also well recognised that isomerism of the atrial

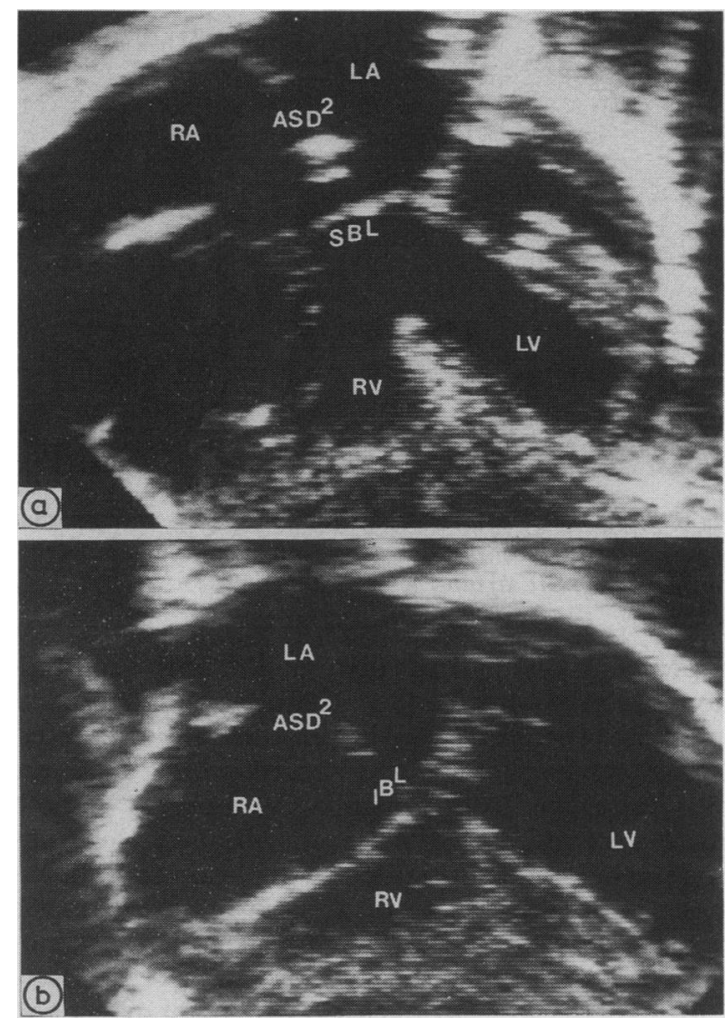

Fig 4 Atrioventricular septal defect with usual atrial arrangement and right hand pattern topology. Note also a secundum atrial septal defect $\left(A S D^{2}\right) ; R A$, right atrium; $L A$, left atrium; $R V$, right ventricle. $L V$, left ventricle; $S B L$, superior bridging leaflet; IBL, inferior bridging leaflet. 


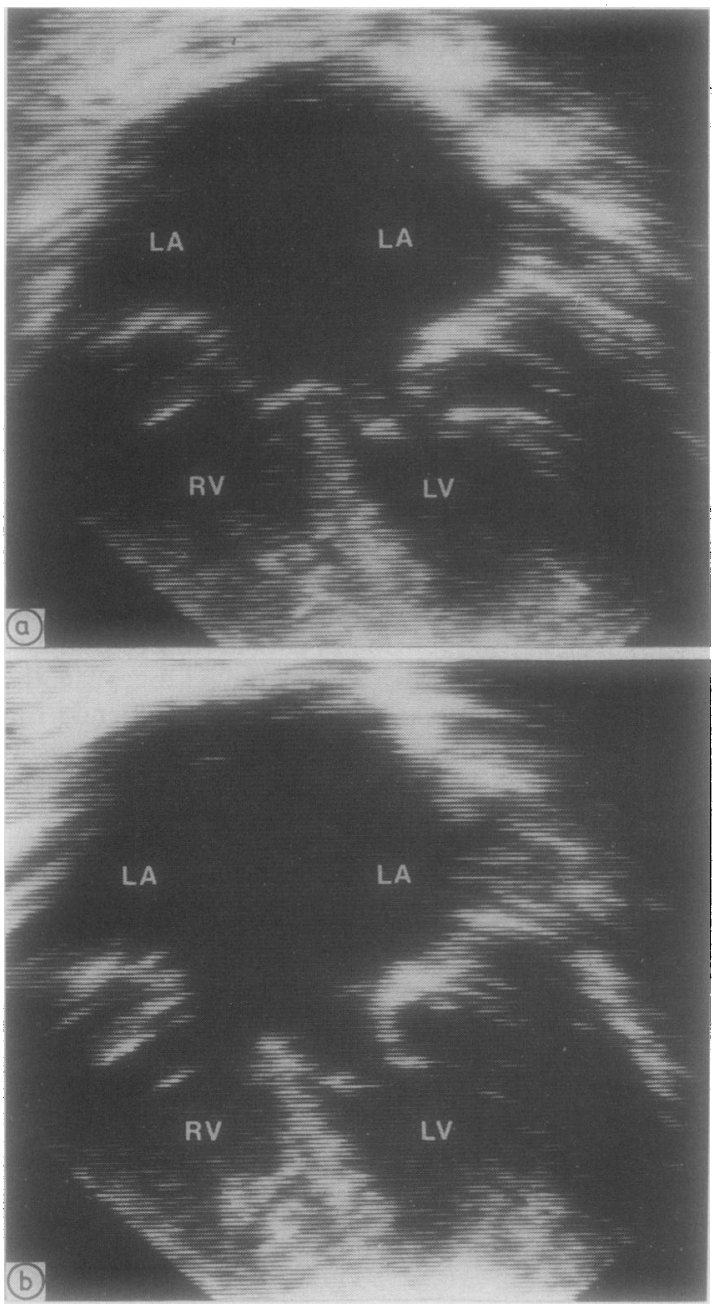

Fig 5 Atrioventricular septal defect with left isomerism and two separate atrioventricular valves with right hand pattern topology. $L A$, left atrium; $R V$, right ventricle; $L V$, left ventricle.

appendages disturbs the distribution of the conduction tissues. So the sinus node is abnormal irrespective of the other associated intracardiac abnormalities. There are two sinus nodes seen when there are bilateral right atrial appendages, whereas if both atrial appendages are of left morphology the sinus node, when present, will be hypoplastic and abnormally located in the lateral atrial wall, either on the right or left side. ${ }^{3}$ The arrangement of the atrioventricular junction is also abnormal.

In patients with atrioventricular septal defect and concordant connections (with usual or mirror image arrangement) the atrioventricular node is inferiorly displaced and lies in the posterior atrial wall beneath the orifice of the coronary sinus. The penetrating bundle is also displaced posteriorly and lies in the
Carvalho, Rigby, Shinebourne, Anderson $\underset{\frac{\Phi}{T}}{\stackrel{\oplus}{\Phi}}$

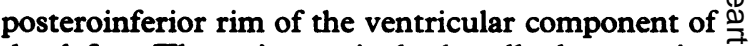
the defect. The atrioventricular bundle then contin- $c$ ues in an exposed position either along the crest or on $\Rightarrow$ the morphologically left ventricular aspect of the $\stackrel{?}{?}$ interventricular septum ${ }^{45}$ where it is at risk of trauma during surgical correction. With right hand topology therefore, sutures should be placed to the right side of $\frac{\bar{\omega}}{T}$ the ventricular septum or to valve tissue on the right, $\mathbb{\AA}$ whereas with left hand topology and mirror image 0 arrangement sutures should be placed on the left side (morphologically right) of the septum.

When there is atrial isomerism and an atrioven- $\overrightarrow{\vec{\omega}}$ tricular septal defect the main determinant of the $\stackrel{S}{\circ}$ distribution of the ventricular conduction tissues is the spatial orientation of the ventricular mass. Right $\rightarrow$ hand topology is always associated with a normal $\vec{\omega}$ posterior atrioventricular node. With left hand pat- $N_{\infty}$ tern, paired atrioventricular nodes are usually or present and there is a well defined sling of specialised $\frac{}{\partial}$ conduction tissue between them. The sling, which may or may not connect the two atrioventricular nodes, runs on the inferior rim of the ventricular septal defect on the morphologically left side of the interventricular septum. The bundle branches arise $\mathscr{\odot}$ from this sling. This again would be a reason for $\subseteq$ always placing sutures on the (morphologically) right ventricular aspect of the interventricular septum.

Dickinson et al reported a case of right atrial isomerism in which there was a second anterior atrioventricular node despite a right hand topological pattern. ${ }^{3}$ Thus while a regular conduction is to be expected when there is an ambiguous atrioven- $\frac{9}{3}$ tricular connection and right hand ventricular $\vec{\partial}$ topology, and an anterior node and/or sling is usual with left hand topology, too few cases have been studied to establish anything more than guidelines. They do, none the less, confirm the crucial role of the concept of ventricular topology in these malformations.

\section{References}

1 Van Praagh R, David I, Gordon D, Wright GB, Van $D$ Praagh S. Ventricular diagnosis and designation. In: Godman MJ, ed. Paediatric cardiology. Vol 4. World N Congress 1980. Edinburgh: Churchill Livingstone, 1981:153-68.

2 Huhta JC, Smallhorn JF, Macartney FJ. Two dimen- N sional echocardiographic diagnosis of situs. Br Heart 0 $J$ 1982;48:97-108.

3 Dickinson DF, Wilkinson JL, Anderson KR, Smith A, Ho SY, Anderson, RH. The cardiac conduction $\mathbb{\&}$ system in situs ambiguus. Circulation 1979;59: 879-85.

4 Thiene G, Anderson RH. The conducting tissues in atrioventricular canal malformations. In: Anderson $\stackrel{\odot}{\Phi}$ RH, Shinebourne EA, eds. Paediatric cardiology. Edinburgh: Churchill Livingstone, 1978:437-47.

5 Kirklin JW, Barrat-Boyes BG. Cardiac surgery. New York: John Wiley \& Sons, 1986:555-9. 